\begin{tabular}{|c|l|}
\hline Title & $\begin{array}{l}\text { Optical Sum-Frequency Emission from Langmuir-Blodgett Films of V ariable Thickness : Effects of the Substrate and } \\
\text { Polar Orientation of Fatty A cids in the Films }\end{array}$ \\
\hline Author(s) & Nishida, Takuma; Johnson, C. Magnus; Holman, Jasper; O sawa, Masatoshi; Davies, Paul B.; Y e, Shen \\
\hline Citation & $\begin{array}{l}\text { Physical Review Letters, 96, 077402 } \\
\text { https://doi.org/10.1103/PhysRevLett.96.077402 }\end{array}$ \\
\hline Issue Date & 2006-02-23 \\
\hline Doc URL & http://hdl.handle.net/2115/5853 \\
\hline Rights & Copyright $\odot 2006$ A merican Physical Society \\
\hline Type & article \\
\hline File Information & PRL96.pdf \\
\hline
\end{tabular}

Instructions for use 


\title{
Optical Sum-Frequency Emission from Langmuir-Blodgett Films of Variable Thickness: Effects of the Substrate and Polar Orientation of Fatty Acids in the Films
}

\author{
Takuma Nishida, C. Magnus Johnson, ${ }^{*}$ Jasper Holman,${ }^{\dagger}$ Masatoshi Osawa, Paul B. Davies, ${ }^{\dagger}$ and Shen $\mathrm{Ye}^{*}$ \\ Catalysis Research Center, Hokkaido University, Sapporo 001-0021, Japan
}

(Received 14 June 2005; published 23 February 2006)

\begin{abstract}
The nonlinear optical response arising from a model multilayer structure, i.e., Langmuir-Blodgett (LB) films comprised of different numbers of per-protonated $(\mathrm{H})$ and per-deuterated (D) fatty acid layers on solid substrates, has been evaluated by sum-frequency generation (SFG) spectroscopy. The SFG signals depend significantly on the absolute polar orientation of the fatty acids in the individual layers and on the nonlinear optical response of the substrate. The SFG spectra on gold and fused quartz substrates demonstrate a totally different dependence on the number of the contributing $\mathrm{H}$ layers, which it is possible to analyze quantitatively. The results provide important information for understanding the origin of the nonlinear optical responses from ordered systems composed of multiple interfaces and therefore for extracting exact structural information about each interface from the observed SFG signals.
\end{abstract}

PACS numbers: 78.67.Pt, 42.65.-k, 68.47.Pe

Ultrathin organic films are the fundamental molecular building blocks of cell membranes, bilayers, and vesicles and consequently of great interest to physicists and biologists alike [1]. They are also the essential components of many nanotechnologies. For example, it is possible to control the size and shape of nanoparticles by using a fatty acid film as a restraining matrix. Although it is believed that the functionalities of organic films depend significantly on their interfacial molecular structures, there is a paucity of such structural information available due to a lack of effective experimental probes, particularly ones capable of operating under ambient conditions. The inherent structural information available from vibrational spectra, hitherto obtained from IR or Raman spectroscopy, has been greatly extended by the introduction of sumfrequency generation (SFG), a 2nd-order nonlinear optical process in which two laser beams at frequencies $\omega_{1}$ and $\omega_{2}$ impinge on a medium, mix and generate a sum-frequency output at $\omega_{s}=\omega_{1}+\omega_{2}$ [2]. Usually, vibrational SFG spectrum is achieved using a fixed frequency visible laser beam $\left(\omega_{1}\right)$ and a tunable frequency infrared laser beam $\left(\omega_{2}\right)$. The intensity of SFG is enhanced when $\omega_{2}$ coincides with a vibrational transition of the interfacial species. By this means a vibrational spectrum of the interface is obtained upshifted into the visible or UV region of the spectrum. SFG is forbidden in a bulk medium with inversion symmetry and only occurs at a surface or interface where the inversion symmetry is necessarily broken. As a surface sensitive probe, SFG offers several advantages over traditional infrared and Raman spectroscopic techniques due to its extremely high surface specificity and sensitivity $[2,3]$. Consequently, it is now widely employed for investigating the molecular structure of various surfaces and interfaces comprised of organic thin films such as selfassembled monolayers (SAMs), Langmuir-Blodgett (LB) films, as well as thin polymer films [4-8]. Although a very powerful technique, the analysis of SFG spectra becomes complicated when several interfaces exist in the same system, which might occur in an organic multilayer in a biological system such as lung surfactant, since more than one of them may be SFG active. The interpretation is further complicated when different supporting substrates are used because these can have nonresonant responses of different magnitudes depending on the electromagnetic interaction between the laser light and the substrate [911]. Our fundamental understanding of the nonlinear optical response from multiple interface systems is still very limited and the development of a rigorous physical model is an essential prerequisite if the technique is to be meaningfully used for more complex systems.

We have recently succeeded in using SFG to probe the "bulk" molecular structure of a LB multilayer by burying one-layer of per-protonated arachidic acid (H) at different locations in a per-deuterated (D) multilayer film on a gold substrate $[8,12-14]$. In the present study, in order to provide a quantitative understanding of how the SFG signals from a multiple interface system arise, LB multilayers were constructed with varying numbers of $\mathrm{H}$ layers, each with the same polar orientation buried in otherwise D layers (Fig. 1) deposited on substrates with both high and low susceptibilities. We report results here on gold and fused quartz substrates representing systems with high and low nonlinear susceptibilities. It was found that the SFG responses from the $\mathrm{H}$ layers within the multilayer film depended significantly on their polar orientation and on the substrate and showed a totally different dependence on the number of $\mathrm{H}$ layers in films on gold compared with films on fused quartz substrates. We have succeeded in quantitatively analyzing these observations.

The samples were prepared on gold or fused quartz substrates by transferring a monolayer of arachidic acid onto the substrate from a $0.3 \mathrm{mM} \mathrm{NaHCO}$ aqueous subphase containing $0.2 \mathrm{mM} \mathrm{CdCl}_{2}\left(p \mathrm{H}, 6.6,20^{\circ} \mathrm{C}\right)$ at $30 \mathrm{mN} \mathrm{m}^{-1}$ by the Langmuir technique $[14,15]$. SFG char- 

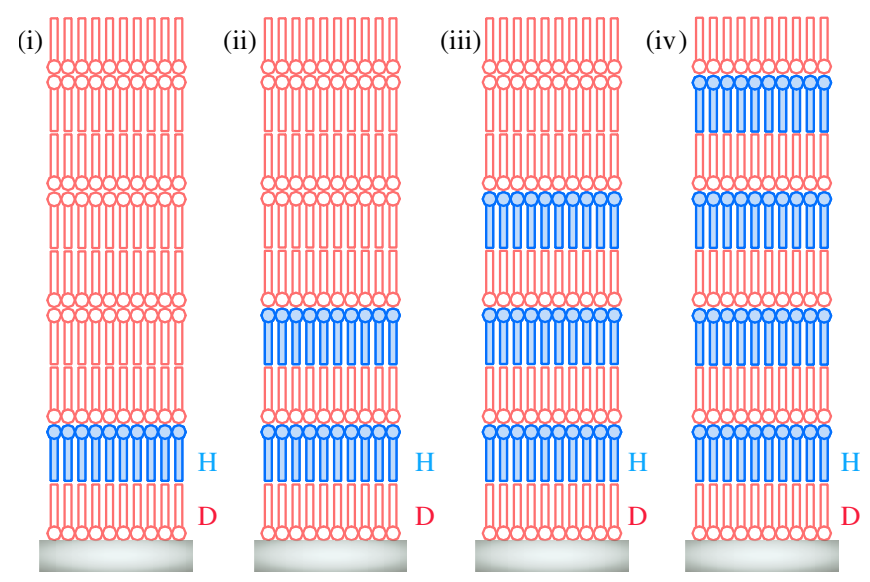

FIG. 1 (color online). Tailored LB multilayers of perdeuterated fatty acid (D) containing (i) 1, (ii) 2, (iii) 3, and (iv) 4 layers of per-protonated fatty acid $(\mathrm{H})$ with the same polar orientation on a solid substrate.

acterizations were carried out in air using a tunable femtosecond broadband infrared pulse $(2.5-10 \mu \mathrm{m})$ and a narrowband picosecond visible pulse $(800 \mathrm{~nm})$ with a copropagating beam geometry $[14,15]$. The $p p p$ combination of SFG, visible, and infrared polarizations was used for the gold substrate and $s s p$ for the fused quartz substrate. The ssp polarization was chosen here for fused quartz since it yields more direct structural information than the $p p p$ combination [16], without prejudicing any of the conclusions reached in this Letter.

Figure 2 shows SFG spectra (squares) recorded in the C$\mathrm{H}$ stretching region (2800-3000 $\mathrm{cm}^{-1}$ ) of H/D nine-layer films on gold (left panel) and fused quartz (right panel), containing one, two, three, or four $\mathrm{H}$ layers, all with their methyl groups pointing towards the substrate, buried in otherwise D layers (Fig. 1). The SFG spectra of the films on the gold substrate give rise to three peaks at 2875, 2935, and $2960 \mathrm{~cm}^{-1}$ which can be attributed to symmetric, Fermi resonance, and asymmetric $\mathrm{C}-\mathrm{H}$ stretching resonances, respectively, of the terminal $\mathrm{CH}_{3}$ group in the $\mathrm{H}$ layers $[14,15]$. The resonance positions are almost identical for the films with different $\mathrm{H}$ layer thicknesses, suggesting that the chemical environments around the $\mathrm{H}$ layers in the film are very similar. Peaks from the chain $\mathrm{CH}_{2}$ groups around 2850 and $2920 \mathrm{~cm}^{-1}$ are very weak in the single $\mathrm{H}$ layer system but become more noticeable in the higher number $\mathrm{H}$ layer films, indicating that some gauche defects exist in the latter. All SFG peaks observed for the $\mathrm{H}$ layers in the multilayers deposited on gold as depicted in Fig. 1 show this upward pointing shape (Fig. 2). Conversely, if the $\mathrm{H}$ layers are deposited with the opposite polar orientation, i.e., methyl groups pointing away from the gold substrate, the SFG resonances become dips (results not shown here), demonstrating that the SFG spectra on gold are very sensitive to molecular orientation and hence the absolute polar orientation of the molecules on the gold substrate can be directly determined $[8,9,12,13]$. On the other hand, ss p-polarized SFG spectra on the fused quartz substrate give rise to two peaks, corresponding to the symmetric and Fermi resonance methyl modes, respectively $[15,16]$. In contrast to the SFG spectra on gold, it should be emphasized here that the resonance peaks on fused quartz always appear as peaks, even from $\mathrm{H}$ layers with a reversed polar orientation [8].

A further important feature is that the SFG intensities observed on both substrates clearly increase with the number $(\boldsymbol{n})$ of $\mathrm{H}$ layers deposited in the multilayer films (Fig. 2). Figure 3 shows the SFG intensities of the symmetric stretching mode of the $\mathrm{CH}_{3}$ group in the multilayer as a function of $\boldsymbol{n}$ on gold (squares) and fused quartz (circles) substrates. The SFG intensities here were normalized to that of a single $\mathrm{H}$ layer on each substrate. In order to compare the dependence on $\boldsymbol{n}$, two dotted lines showing linear (blue) and quadratic (red) functions of $\boldsymbol{n}$ are superimposed on the data in the figure. It is clear that the SFG intensities observed here follow a quite different dependence on $\mathrm{H}$ layer number $\boldsymbol{n}$ with a stronger dependence on a fused quartz substrate than that on a gold substrate (Fig. 3).

Generally, the SFG intensity $\left(I_{\mathrm{SFG}}\right)$ of the $\mathrm{CH}_{3}$ group in a multilayer containing $\boldsymbol{n}$ layers of $\mathrm{H}$ can be described as $[2,3]$

$$
I_{\mathrm{SFG}} \propto\left|\chi_{1}^{(2)}+\chi_{2}^{(2)}+\cdots+\chi_{n}^{(2)}+\chi_{\mathrm{NR}}^{(2)}\right|^{2},
$$

where $\chi_{\mathrm{NR}}^{(2)}$ is the nonresonant contribution from the substrate and $\chi_{n}^{(2)}$ represents the 2nd-order susceptibility for the $n$th $\mathrm{H}$ layer (counting up from the substrate) which can be described as $[2,3]$

$$
\chi_{n}^{(2)}=\sum_{q} \frac{A_{q}}{\omega_{\mathrm{IR}}-\omega_{v, q}+i \Gamma_{q}},
$$

where $A_{q}, \omega_{v, q}$, and $\Gamma_{q}$ are the strength, frequency, and damping factor, respectively, of the $q$ th vibrational mode and $\omega_{\mathrm{IR}}$ is the infrared frequency. In the present experiment, we have deliberately fabricated a multilayer structure where all $\mathrm{H}$ layers have an identical polar orientation, i.e., $\chi_{1}^{(2)}=\chi_{2}^{(2)}=\chi_{3}^{(2)}=\cdots=\chi_{n}^{(2)}$, and so on. Hence, Eq. (1) can be simplified to

$$
I_{\mathrm{SFG}} \propto\left|n \chi_{n}^{(2)}+\chi_{\mathrm{NR}}^{(2)}\right|^{2} .
$$

It can be anticipated from Eq. (3) that the observed SFG intensities should directly associate with the $\mathrm{H}$ layer number $(\boldsymbol{n})$ in the multilayer as observed in Figs. 2 and 3. On the other hand, no thickness dependence was observed in previous SFG spectra for the isotropic multilayer only composed of $H$ layers $[14,15]$. This is to be expected since the bilayer structure in the isotropic multilayer film shows a reversed polar orientation so $\chi_{1}^{(2)}=-\chi_{2}^{(2)}, \chi_{3}^{(2)}=-\chi_{4}^{(2)}$, etc. Therefore, SFG intensity only arises from the unique topmost layer. Furthermore, the SFG intensity and the 

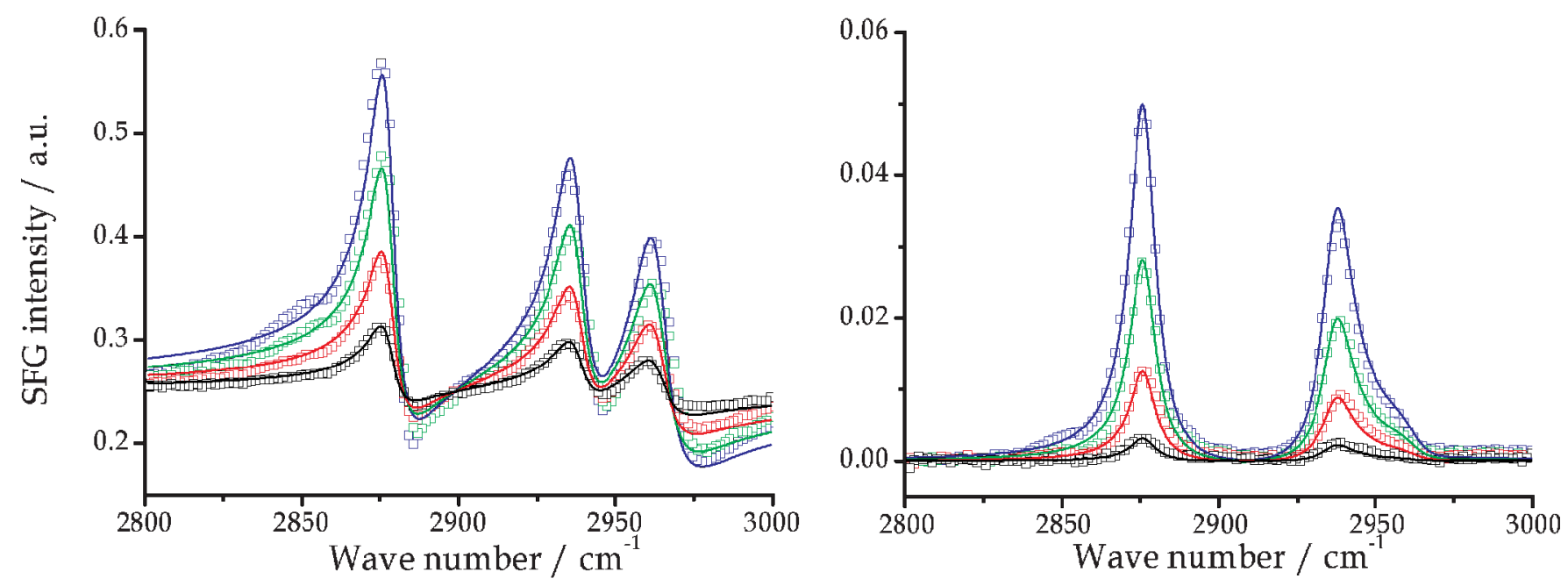

FIG. 2 (color). SFG spectra (square symbols) of LB multilayers of per-deuterated arachidic acid containing 1- (black), 2- (red), 3- (green), and 4- (blue) per-protonated layers on gold (left) and fused quartz (right) with the layer scheme given in Fig. 1. The solid line of the same color corresponds to the fitted (1-layer) and simulated (2-, 3-, and 4-H) results using Eqs. (3) and (2).

spectral line shape are directly dependent on the relative magnitude and phase of the resonant susceptibility $\left(\chi_{n}^{(2)}\right)$ in comparison with those of the nonresonant contribution $\left(\chi_{\mathrm{NR}}^{(2)}\right)$ [Eq. (3)]. In order to quantitatively evaluate the $\boldsymbol{n}$-dependent SFG intensity observed on the different substrates (Figs. 2 and 3), Eq. (3) can be rearranged and expressed in terms of the SFG intensity for a single $\mathrm{H}$ layer system $\left(I_{\mathrm{SFG}, 1}\right)$ and $\mathrm{H}$ layer number $\boldsymbol{n}$,

$$
\begin{aligned}
I_{\mathrm{SFG}} & \propto n \times I_{\mathrm{SFG}, 1}+K \times\left[n(n-1)\left|\chi_{n}^{(2)}\right|^{2}-(n-1)\left|\chi_{\mathrm{NR}}^{(2)}\right|^{2}\right] \\
& \propto n^{2} \times I_{\mathrm{SFG}, 1}+K \times\left[-n(n-1)\left(\chi_{n}^{(2)} \chi_{\mathrm{NR}}^{(2)^{*}}+\chi_{n}^{(2)^{*}} \chi_{\mathrm{NR}}^{(2)}\right)-\left(n^{2}-1\right)\left|\chi_{\mathrm{NR}}^{(2)}\right|^{2}\right],
\end{aligned}
$$

where $*$ corresponds to the complex conjugate and $K$ is a constant representing the contribution from Fresnel factors and excitation laser power densities, etc. [2,3]. It should be emphasized that Eqs. (4a) and (4b) are completely equivalent to each other and are presented in alternative forms

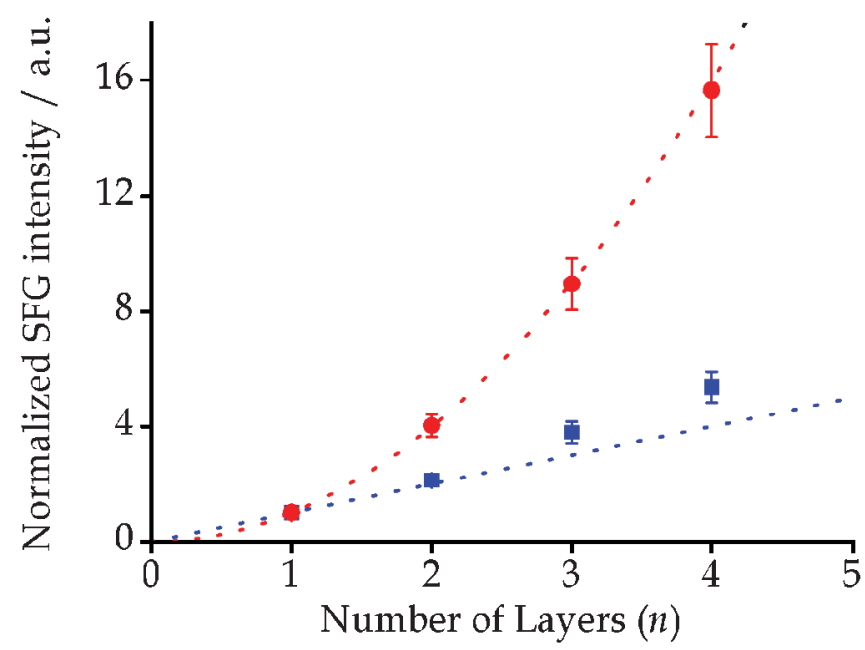

FIG. 3 (color). Normalized SFG intensities of $\nu_{\text {sym }}\left(\mathrm{CH}_{3}\right)$ of LB multilayer systems of arachidic acid shown in Fig. 2 on gold (squares) and fused quartz (circles) substrates. See text for details. merely to facilitate the discussion. In fact, the above relation can be further simplified depending on the relative values of $\chi_{n}^{(2)}$ and $\chi_{\mathrm{NR}}^{(2)}$.

If $\chi_{\mathrm{NR}}^{(2)} \approx 0$, i.e., the case when fused quartz, which has a negligible nonresonant background under the present laser excitation conditions, is used as a substrate, Eq. (4b) can be truncated to

$$
I_{\mathrm{SFG}} \propto n^{2} \times I_{\mathrm{SFG}, 1},
$$

i.e., the total SFG intensity of a multilayer containing $\boldsymbol{n}$ per-protonated layers, which having identical polar orientation on fused quartz is expected to be $\boldsymbol{n}^{2}$ times that of a single per-protonated layer. This is confirmed by the results in Figs. 2 and 3. Since there is a negligible nonresonant background [Eq. (5)], SFG spectra on fused quartz substrates will only appear as peaks. On the other hand, the $\chi_{\mathrm{NR}}^{(2)}$ of the gold substrate is larger than, but still comparable to, $\chi_{n}^{(2)}$ for the $\mathrm{CH}_{3}$ group at the present visible or SFG wavelength region $[8,10,11]$, and hence the contribution from the two terms in the square brackets [Eqs. (4a) and (4b)] must be taken into account. Therefore, $I_{\mathrm{SFG}}$ from the $\mathrm{H}$ layers in a multilayer on a gold substrate would be expected to depart from the linear or quadratic relationships. Although a quantitative estimation for the contribution from these terms requires further work, it is never- 
theless the case that the experimental dependence for a gold substrate is much closer to a linear than a quadratic dependence for small $\mathrm{H}$ layer numbers (Fig. 3). The above analysis demonstrates the important role played by the substrate in SFG spectra.

As shown in Fig. 2, the SFG spectra for the multilayers containing two, three, and four $\mathrm{H}$ layers (red, green, and blue lines, respectively) are well reproduced by a simulation employing Eq. (3) based on the spectral parameters determined by fitting the SFG curves of the single $\mathrm{H}$ layer system on both substrates (black lines, Fig. 2). The spectral simulation fits the experimental results well with the exception of some minor discrepancies arising from the $\mathrm{CH}_{2}$ resonances caused by a small number of gauche defects in the single $\mathrm{H}$ layer film (Fig. 2). These results clearly demonstrate that the model employed above is valid and accurate.

In practice evidence for partial mixing between neighboring layers in LB multilayer films comprised of alternating layers of per-deuterated (D) and per-protonated fatty acid $(\mathrm{H})$ has been established by neutron and $\mathrm{x}$-ray reflectometry $[17,18]$ and atomic force microscope (AFM) [19]. However, in the present experiment it was found that in films comprised of both per-deuterated and per-protonated layers the SFG intensities remained constant over a period of months after preparation. This observation is in agreement with the reports that the intermixing between the layers takes place during the initial deposition process [17-19]. Although the absolute SFG intensity for each $\mathrm{H}$ layer is expected to decrease due to the decrease in asymmetry caused by the H/D layer intermixing, the quantitative relationships given above should remain valid if one assumes that the same ratio of $\mathrm{H}$ and $\mathrm{D}$ molecules exchange during the sample preparation. This is a reasonable assumption since the simulations fit the experimental results fairly well (Fig. 2). Further investigations of these effects are under way.

In summary, we have demonstrated the effects of the polar orientation and of the substrate on the nonlinear optical response from a well-defined H/D multilayer. These effects can be analyzed quantitatively even when a partial intermixing of H/D molecules in adjacent layers takes place. Greater layer number dependence of the SFG signal can be obtained from a substrate with a negligible nonresonant susceptibility (fused quartz) than that from a substrate with a large nonresonant susceptibility (gold). These results provide a quantitative understanding of the origin of the nonlinear responses from ordered systems containing multiple interfaces.

This work was partially supported by JST (PRESTO and CREST). S. Y. gratefully acknowledges support from Min- istry of Education, Culture, Sports, Science, and Technology (MEXT) of the Japanese Government (Scientific Research for Priority Areas 417, No. 17029003) and a grant from the Nippon Sheet Glass Foundation for Materials Science and Engineering. C. M. J. acknowledges financial support from the Sweden-Japan Foundation. J. H. and P. B. D. thank the Japan Society for the Promotion of Science (JSPS) for financial support.

*Also at Division of Corrosion Science, Royal Institute of Technology, Stockholm, Sweden.

${ }^{\dagger}$ Also at Department of Chemistry, University of Cambridge, Cambridge, United Kingdom.

Electronic address: ye@cat.hokudai.ac.jp

[1] A. Ulman, An Introduction to Ultrathin Organic Films (Academic, New York, 1991).

[2] Y.R. Shen, The Principles of Nonlinear Optics (Wiley, New York, 1984).

[3] Y. R. Shen, Proc. Natl. Acad. Sci. U.S.A. 93, 12104 (1996).

[4] C.D. Bain, J. Chem. Soc., Faraday Trans. 91, 1281 (1995).

[5] P. B. Miranda and Y.R. Shen, J. Phys. Chem. B 103, 3292 (1999).

[6] G. L. Richmond, Chem. Rev. 102, 2693 (2002).

[7] Z. Chen, Y.R. Shen, and G. A. Somorjai, Annu. Rev. Phys. Chem. 53, 437 (2002).

[8] J. Holman, P. B. Davies, T. Nishida, S. Ye, and D. J. Neivandt, J. Phys. Chem. B 109, 18723 (2005).

[9] R. N. Ward, P. B. Davies, and C. D. Bain, J. Phys. Chem. 97, 7141 (1993).

[10] M. A. Hines, J. A. Todd, and P. Guyot-Sionnest, Langmuir 11, 493 (1995).

[11] T. Ishibashi and H. Onishi, Appl. Phys. Lett. 81, 1338 (2002).

[12] J. Holman, P.B. Davies, and D. J. Neivandt, J. Phys. Chem. B 108, 1396 (2004).

[13] J. Holman, S. Ye, P. B. Davies, and D. J. Neivandt, J. Am. Chem. Soc. 126, 14322 (2004).

[14] S. Ye, H. Noda, S. Morita, K. Uosaki, and M. Osawa, Langmuir 19, 2238 (2003).

[15] S. Ye, H. Noda, T. Nishida, S. Morita, and M. Osawa, Langmuir 20, 357 (2004).

[16] P. Guyot-Sionnest, J.H. Hunt, and Y.R. Shen, Phys. Rev. Lett. 59, 1597 (1987).

[17] D. G. Wiesler, L. A. Feigin, C. F. Majkrzak, J. F. Ankner, and V. I. Troitsky, Thin Solid Films 266, 69 (1995).

[18] U. Englisch, T. A. Barberka, U. Pietsch, and U. Hohne, Thin Solid Films 266, 234 (1995).

[19] D. Y. Takamoto, E. Aydil, J. A. Zasadzinski, D. K. Ivanova, A. T. Schwartz, T. Yang, and P.S. Cremer, Science 293, 1292 (2001). 